\title{
Study of Healthcare Annotation Systems
}

\author{
Khalil Chehab, Faculty of Economics and Management, Sfax, Tunisia \\ Anis Kalboussi, Higher Institute of Computer Science and Management, University of Kairouan, Tunisia \\ Ahmed Hadj Kacem, Faculty of Economics and Management, Sfax, Tunisia
}

\begin{abstract}
The annotation practice is an almost daily activity used by healthcare professionals (PHC) to analyze patients' records, collaborate, share knowledge, and communicate. These annotations are generated within a healthcare cycle. Similarly, this cycle represents the life cycle of annotations in the patient record. The exponential increase in the number of medical annotation systems made the choice of a system by a PHC difficult, in a well-defined context (biology, radiology) and according to his/her needs to the functionalities offered by these tools. Therefore, the authors propose two taxonomies to distinguish annotation tools developed by industry and academia over the last two decades. The first classification provides an external vision based on five generic criteria. The second classification is an internal vision that gives us an idea about the functionalities offered by these systems. Finally, these unified and integrated classifications criteria are used to organize and observe the limitation of 50 medical annotation tool systems.
\end{abstract}

\section{KEYWORDS}

Annotation, Annotation System, Classification, Competency, E-Health, Health Record, Healthcare Cycle, Healthcare Processes, Healthcare Professionals, Web Service

\section{INTRODUCTION}

The paper annotation practice is very common. Indeed, during our reading, we are all accustomed to writing our comments in the margin of the document, highlighting or circling part of the text to enrich and add value to information (Khalil Chehab, Kalboussi, \& Kacem, 2019; Anis Kalboussi, Mazhoud, Omheni, \& Kacem, 2014; Anis Kalboussi, Omheni, Mazhoud, \& Kacem, 2015a). Annotation is a central practice in many professions: teachers annotate copies of students; professors' exchange annotated documents during their work; engineer co-builds engines by annotating sketches of plans to make them evolve, doctors' comment on the patient records...etc. (K. Chehab, Kalboussi, \& Kacem, 2018; A. Kalboussi, Mazhoud, Hadj Kacem, \& Omheni, 2013; Anis Kalboussi, Mazhoud, \& Kacem, 2016b). The practice of pencil-and-paper annotation, among healthcare professionals (PHC) in the patient record is common and contributes to the enrichment of knowledge and skills of health professionals (PHC). With the emergence of new information and communication technologies, the field of digital health (Dingli \& Seychell, 2014; Yu \& Yilayavilli, 2010), also called e-health, has undergone a significant and promising technical revolution over the last decade (Charlet et al., 1998). This revolution allowed health professionals to take full advantage of the computerized medical services by sharing the important data, the necessary knowledge and the key skills in a digital world (Anis Kalboussi, Mazhoud, \& Kacem, 2013). Among the applications that are covered by this digital revolution, there are the annotation systems (Dutta, Mondal, \& Paul, 2020; Kraljevic et al., 2019; Ma 
\& Meng, 2018; Philbrick et al., 2019; Segura et al., 2017) dedicated to healthcare professionals (local application/web application/plug-in) that have been developed to manage digitally the annotations of these latter. Here, annotations can cover different domains of healthcare and can have different types. Moreover, they can be private, public, or shared, according to the annotated content (Bringay, Barry, \& Charlet, 2004). As a result, these systems are becoming increasingly claimed and felt by the different medical actors, hence, they offer the necessary means to explain and enrich the information with personal observations and permit ideas sharing which in turn improve collaborative working practices.

In this work, we started with an exhaustive reading for the available papers on annotation systems in general and medical annotation systems in particular. Although the medical annotation systems have already been studied in a variety of contexts (doctor, nurse, biologist, radiologist), yet when it comes to the PHC to choose which system to use based on the functionalities offered by these latter is difficult. Also it is not a trivial task for a researcher he wants to identify future research areas based on existing annotation systems. This is because the annotation systems are so common and many of them share similar objectives which can either be to create annotations or to manipulate them with fairly classic functionalities. Moreover, there are no formal criteria to facilitate the comparison between those systems and to guide PHC choice or a researcher. As a result, there is a fragmented picture of these annotation tools. As far as we know, this is the first work to consider the classification of medical annotation system. The purpose of this paper is to provide a unified idea about the annotation systems used by healthcare professionals. This panoramic view is based on a classification of fifty different annotation systems developed in literature over the past two decades by industry and researchers. The concentration of the difference permits to conclude the classifications criteria and to highlight the challenges in this research field. In this paper, first, the organization of annotation systems is built on the basis of five generic criteria (Azouaou, 2006; Anis Kalboussi, Omheni, Mazhoud, \& Kacem, 2015b): standard annotation (computational / cognitive); category of the annotation system (application / plug-in / website); type of annotative activity (manual / semi-automatic / automatic); annotated resource type (text / video / image / doc / HTML / pdf/ etc.) and practitioner that is a PHC. A second comparison is made based on the functionalities offered by the medical annotation systems. Moreover, based on the extensive review of the existing annotation systems we identify the limitation according to the defined criteria.

The rest of the paper is organized as follows: Section 2 gives a general presentation of the annotation systems and a classification of these tools based on several criteria; Section 3 draws some key observations and a discussion of open research problems on annotation systems. Finally, section 4 concludes this paper.

\section{ANNOTATION IN MEDICAL SYSTEMS}

In the literature, there are several definitions of medical annotation. For example, in (Bringay et al., 2004) health record annotation is seen as "a particular note related to a target. The target can be a collection of documents, a document, a segment of the document (a paragraph, a group of words, an image, part of an image, etc.) or an annotation. Each annotation has content, materialized by an inscription. This is the trace of a mental representation elaborated by the annotator about the target, resulting from a cognitive process located, reading the annotated document. The content of the annotation may be interpreted by another reader. The anchor links the annotation to the target (an arrow, a circled sentence, etc.).”. In (Anis Kalboussi et al., 2015b), the biomedical annotation is defined as "a biological interpretation, an enrichment of knowledge sharing in the life sciences. We divide protein sequence information into inflexible data called basic data and data that characterize sequences so called annotation". Based on the definitions cited above and more (Anis Kalboussi et al., 2015b), we conclude that the creation of an annotation is composed of two main parts. (i) The activity, the realization of user objectives (implicit or explicit) as highlight information, request help, to share in case of digital support...etc. based on the document in hand. (ii) The object, which considered as 
the result of an activity. Here, it refers to shapelform the user draw to highlight pieces of information i.e., circle, underline, square...etc. and the extra information added by him lher. It worth mentioning that this extra information falls in the same context as the original information and had been added with the goal to enrich not to modify. To this end, we can say that the activity is the creation process of an object on a supportldocument. In follow, we detail each part:

\section{THE ANNOTATION OBJECT}

\subsection{Object Components}

- Anchor: The anchor of the annotation can relate to different types of passages, a single word, a line, a paragraph or an entire document. (Azouaou, 2006; Mille, 2005; Veron, 1997) distinguishes several levels of the anchor, where he considers that the annotation is: in the document (on the page, between the pages) or outside the document. We can globally distinguish two types of annotations according to whether their anchor is inside the document or outside: internal annotations and external annotations;

- Graphical form: The different forms that annotations take on the document are particularly studied by the authors. (Mille, 2005) makes a very exhaustive review of the different graphic forms identified in the literature. Mille first takes up the categorizations proposed by the other authors and then proposes a more complete ontology of possible annotation act (addition of the text, highlighting, etc).

\subsection{Object Structure}

Formalizing the annotation object, regardless of the annotation action, amounts to defining the composition of its structure, (i) what it contains, and (ii) the properties that make it possible to identify and describe it i.e., location of an object in the document, objective of the annotation, author, creation date, type...etc. In the literature, there are different models (Denoue, 2000; Kahan \& Koivunen, 2001; Veron, 1997; Weibel, 1997) agree on some fundamental properties: such as the author, the date of creation, the semantics (purpose of the annotation) or the content...etc. where the rest of the properties depends on the needs for which these models were designed.

\subsection{The Annotation Activity}

After studying the annotation object, in this section, we focus on the activity i.e., creation of an annotation object also called "annotation". This activity consists of placing graphic forms on the document. Authors in (Huart, 1996; Marshall, 1997), studied the relations correspondence (annotation practices) between a set of graphical forms and the objective (semantic) of the annotation. These relations of correspondence are on one side common and shared in a group and which are on the other hand different according to the context of the annotation. The study demonstrated that it is possible to deduce the goal of the annotations from their graphical form in a context given.

\subsection{Features of Medical Annotation Systems}

Several medical annotation systems have been developed, in recent years, in the context of digital health domain evolution; these systems are created based on several divergent characteristics (comes from the existence of several standards) related to the concept of annotation and the developed system. An annotation system can be developed as plug-in, web application, or local application. The system is used by a practitioner who is a PHC (doctor, nurse, biologist, and radiologist). The annotation activity style on a system is either: automatic, manual, or semi-automatic. As described above, the annotation activity results in an annotation object of type cognitive or computational. Clearly this assemble three main ideas: 1) the system used to create this annotation hence, each system type has its objectives; 2) what composed the annotation itself as explained above and on what had been 
created; 3) the creator of the annotation because each PHC has a certain role and access constraints within a particular medical system. Eventually, those characteristics can serve as criteria to classify the medical annotation systems. In follow, we discuss in-depth independently each of these criteria:

\subsection{Type of Medical Annotation Object (Cognitive / Computational)}

- Cognitive Annotation Object: Has a visible visual form on the digital resources (text, image, video, etc.) and created to be used and analyzed by a human agent. In this case, the annotation requires a cognitive and intellectual effort to be interpreted. The cognitive annotations are regarded as additional content that is strongly related to existing content, meaning that they add values to the existing content by providing an additional layer of elucidation and explanation (Cahier \& Zacklad, 2004). This type of annotation is integrated into the application that is used essentially by the radiologist, doctors;

- Computational Annotation Object: Also known as metadata is intended to be processed and manipulated by software agents. These objects allow users to annotate digital resources like web pages, text files, databases and even for images and videos to facilitate their exploitation by machines. It is used in the field of information retrieval, summarization, document classification, and indexing. This type of annotation is integrated into the applications that are used essentially by biologists in the domain of Bioinformatics.

\subsection{The Medical Annotation Activity (Manual, Automatic, Semi-Automatic)}

Annotation activity begins with the choice of an anchor and an annotation form, from the annotation software toolbar. Here, each annotation has numerous properties such as author, creation date ...etc. This process ends with the attachment of the annotation to a well-defined target. Based on this process, we can classify the annotative activity as manual, automatic or semi-automatic (Khalil Chehab, Kalboussi, \& Kacem, 2020; Anis Kalboussi, Mazhoud, \& Kacem, 2016a).

- Manual: The process already mentioned will be carried out totally by the user himself, who selects the form of the annotation, the anchor and creates the annotation. This process is like the process of annotation when paper support is available. Clean tools ("Clean tools," 2018), IOGram ("IOGram," 2018) and Medip ("Medip," 2018)are examples of the manual annotation systems;

- Automatic: The annotation process is carried out totally by the machine. These annotations are done based on context sensors, pattern recognition techniques...etc. Epvizr (Ma \& Meng, 2018), BioR (Kocher et al., 2014) and BioQRator (Kwon, Kim, Shin, \& Wilbur, 2013) are examples of automatic annotation systems;

- Semi-Automatic: in this case, the process will be done at the start by the user. After a while, the system acquires and understands how the user annotates. In this state, the system suggests annotations automatically, based on an annotation model built with rules under development. At this stage, human intervention remains just to validate or not validate the suggested annotation which in turn helps to refine the annotation rules created at a certain level. When there are no corrections and there is a complete acceptance of the suggested rules, human intervention is no longer considered or needed, and the process becomes totally automated. Medetect (Tian et al., 2013), Flersa (Navarro-Galindo \& Samos, 2012) and Bioannote (López-Fernández et al., 2013) are examples of the semi-automatic annotation systems.

\section{HEALTHCARE PROFESSIONAL (PRACTITIONER)}

It is the annotator that is equipped with an annotation system to use all the functionalities offered by the latter. In our case, the practitioners are healthcare professionals (Doctors, nurses, biologists, and 
radiologists). The healthcare cycle is composed of four phases: diagnostic, treatment, advice, follow up and observation. Each practitioner, with a medical annotation system, intervenes in one or many phases according to their role to accomplish a specific task in which annotation is made.

\section{TYPE OF ANNOTATION SYSTEM (APPLICATION, PLUG-IN, WEBSITE)}

Several annotation systems are created with different architectures and interfaces that permit annotation adding on digital resources. These annotation systems can be classified according to three criteria:

- Application: An application is created to annotate the resources already consulted. These applications offer several functionalities as cited below in section 2.3. Several applications exist such as Heideltime (Zell \& Strötgen, 2015), Biocat (Zhou, Lamichhane, Sterne, Ye, \& Peng, 2013) and Bioannote (López-Fernández et al., 2013);

- Plug-in: These are the expansion modules, an external module that is added to a website or software and which makes it possible to provide annotation functionalities to the latter. Several plug-in exist, such as BioR (Kocher et al., 2014), Domeo annotation (Clark, Ciccarese, \& Goble, 2014) and Cart (Deghou et al., 2016);

- Website: These are specialized websites to annotate consulted resources by registered users on the web. Several websites exist, such as Mammoapplet (Mata, Oliver, Torrent, \& Martí, 2012), DSS-meda (Pawel Mrozowski and Andrzej A. Kononowicz, 2006) and 3dmarkup radiologist (Moreira, Hage, Luque, Willrett, \& Rubin, 2015).

\subsection{Type of Annotated Resource}

Annotated resources can be a word document, pdf, image, text, video, HTML, audio, etc. Table 1 presents a comparative study of fifty medical annotation systems seen in the literature study using the 5 criteria already explained. These annotation systems are ranged on the table according to the chronological order of their publication year. Here, some systems can include manual and automatic annotation treatment or more options thus, we added $(x)$ in all the relative box in the table.

\subsection{Functionalities of Medical Annotation Systems}

The PHC, who is the annotation system user, benefits from the features offered by these systems (Colley, Rantakari, Virtanen, \& Häkkilä, 2017). These systems share several features that are dedicated to annotation management, search, etc. Other features are created especially for a need related to the studied resource, practitioners...etc. In the previous section, we provided a unified classification of annotation systems in e-health domain. In this part, we are interested in making a comparison of these annotation systems based on the offered functionalities offered:

- Annotation Management: The existing annotation systems can be classified into two categories. The first category links an annotation to an entire document by a URL, in which case the anchor of the annotation is the entire document. In the second category, the system associates the annotation to a specific part in a document and which is identified by two elements: (i) the annotated part and (ii) the URL; in this case the anchor of the annotation is the annotated part. Each medical annotation system studied can manage annotations through at least one of these functionalities:

- Creating Annotation: Can be automatic, manual or semi-automatic however; in the healthcare field, the automatic annotation is more popular;

- Modifying Annotation: With this function, we can modify the anchor, the shape, the annotating content, and the annotated content; 
Table 1. Comparative study of the medical annotation systems using 5 criteria

\begin{tabular}{|c|c|c|c|c|c|c|c|c|c|c|c|c|c|}
\hline \multirow{2}{*}{\multicolumn{3}{|c|}{ Name of the medical annotation system }} & \multirow{3}{*}{\begin{tabular}{|l|} 
Year \\
2019 \\
\end{tabular}} & \multirow{3}{*}{ 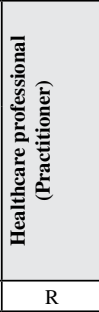 } & \multirow{3}{*}{$\begin{array}{c}\text { Annotated } \\
\text { Resource type }\end{array}$} & \multicolumn{3}{|c|}{$\begin{array}{c}\text { Category } \\
\text { of } \\
\text { annotation } \\
\text { system } \\
\end{array}$} & \multicolumn{2}{|c|}{$\begin{array}{l}\text { Annotation } \\
\text { Type }\end{array}$} & \multicolumn{3}{|c|}{$\begin{array}{c}\text { Type of } \\
\text { annotation } \\
\text { activity }\end{array}$} \\
\hline & & & & & & \multirow[t]{2}{*}{ 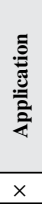 } & \multirow{2}{*}{ 章 } & \multirow[t]{2}{*}{$\stackrel{0}{\equiv}$} & \multirow{2}{*}{ 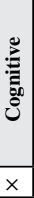 } & \multirow[t]{2}{*}{ 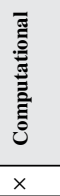 } & \multirow{2}{*}{ 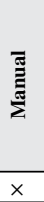 } & \multirow{2}{*}{ 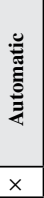 } & \multirow{2}{*}{ 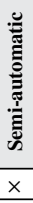 } \\
\hline 1 & RIL-Contour & (Philbrick et al., 2019) & & & & & & & & & & & \\
\hline 2 & Santo & (“Santo," 2018) & 2018 & $\mathrm{D}$ & Text & & & $\times$ & $x$ & & & $x$ & \\
\hline 3 & IOGram & (“IOGram,” 2018) & 2018 & $\mathrm{D}$ & Image 3D & $\times$ & & & $x$ & & $\times$ & & \\
\hline 4 & Crowdflower & (“Crowdflower," 2018) & 2018 & $\mathrm{R}$ & Image & $x$ & & & $\times$ & & $x$ & & \\
\hline 5 & Epivizr & (Ma \& Meng, 2018) & 2018 & $\mathrm{~B}$ & Document & $\times$ & & & $x$ & & $x$ & $\times$ & \\
\hline 6 & Medip & (“Medip," 2018) & 2018 & $\mathrm{R}$ & Image & $x$ & & & $x$ & & $x$ & & \\
\hline 7 & ODMSummary & $\begin{array}{c}\text { (Storck, Krumm, \& Dugas, } \\
\text { 2017) }\end{array}$ & 2017 & $\mathrm{D}$ & HTML & & & $x$ & $x$ & & & $x$ & \\
\hline 8 & 3dBionote & (Segura et al., 2017) & 2017 & $\mathrm{~B}$ & Image & & & $\times$ & & $x$ & $\times$ & & \\
\hline 9 & Verdant & $\begin{array}{c}\text { (McKain, Hartsock, Wohl, \& } \\
\text { Kellogg, 2017) }\end{array}$ & 2017 & B & Text & & & $x$ & & $x$ & & $x$ & \\
\hline 10 & Best slice & (“Best slice,” 2017) & 2017 & $\mathrm{R}, \mathrm{D}$ & Image & & & $\times$ & $\times$ & & $\times$ & & \\
\hline 11 & MicroMD & (“"MicroMD," 2017) & 2017 & $\mathrm{R}, \mathrm{D}$ & Image & $\times$ & & & $x$ & & $x$ & & \\
\hline 12 & Med3D & $\begin{array}{c}\text { (Lavrič, Bohak, \& Marolt, } \\
\text { 2017) }\end{array}$ & 2017 & $\mathrm{D}$ & Image & $\times$ & & & & $x$ & $x$ & & \\
\hline 13 & GIDAC & (Vizza et al., 2017) & 2017 & $\mathrm{R}$ & Image & $x$ & & & $x$ & & $x$ & & \\
\hline 14 & Vcf-miner & (Hart et al., 2016) & 2016 & $\mathrm{~B}$ & Text & & & $x$ & & $x$ & $x$ & & \\
\hline 15 & Plexo & ("Plexo," 2016) & 2016 & $\mathrm{R}$ & Image & & $x$ & & $x$ & & $x$ & & \\
\hline 16 & BioDigital human & (Qualter et al., 2012) & 2016 & $\mathrm{D}$ & Image 3D & $x$ & & & $\times$ & & $x$ & & \\
\hline 17 & Leadtools & (“Leadtools," 2016) & 2016 & $\mathrm{R}$ & Image & & & $\times$ & $\times$ & & $x$ & & \\
\hline 18 & SemAnatomy3D & $\begin{array}{l}\text { (I. Banerjee G. Patané \& } \\
\text { Spagnuolo, 2015) }\end{array}$ & 2015 & $\mathrm{R}$ & Image & $\times$ & & & $\times$ & & $x$ & $x$ & \\
\hline 19 & Icare & $\begin{array}{c}\text { (Marrast, Zaraté, \& Mayère, } \\
\text { 2013) }\end{array}$ & 2015 & $\mathrm{~N}$ & Document & $x$ & & & $x$ & & $x$ & & \\
\hline 20 & Cart & (Deghou et al., 2016) & 2015 & $\mathrm{~B}$ & Image & & $x$ & & & $x$ & $x$ & & \\
\hline 21 & Heideltime & (Zell \& Strötgen, 2015) & 2015 & $\mathrm{~A}$ & Time & $x$ & & & $\times$ & & $x$ & & \\
\hline 22 & Clamp & (“Clamp," 2015) & 2015 & $\mathrm{D}$ & Text & $\times$ & & & $\times$ & & $x$ & & \\
\hline 23 & Perfectcare & ("Perfectcare," 2015) & 2015 & $\mathrm{D}$ & Document & & & $\times$ & $x$ & & $x$ & & \\
\hline 24 & \begin{tabular}{|l|} 
Domeo Annotation \\
\end{tabular} & (Clark et al., 2014) & 2014 & $\mathrm{~B}$ & HTML & & $x$ & & & $x$ & $x$ & $x$ & $x$ \\
\hline 25 & BioR & (Kocher et al., 2014) & 2014 & $\mathrm{~B}$ & Text & & $x$ & & & $x$ & $x$ & $x$ & \\
\hline 26 & $\begin{array}{l}\text { 3dmarkup } \\
\text { radiologist }\end{array}$ & (Moreira et al., 2015) & 2014 & $\mathrm{R}$ & Image & & & $\times$ & & $\times$ & $x$ & & \\
\hline 27 & Vita & (Roy, Brown, \& Shih, 2014) & 2014 & $\mathrm{R}$ & Image, video & $x$ & & & $\times$ & & $x$ & & \\
\hline 28 & Marky & $\begin{array}{c}\text { (Pérez-Pérez, Glez-Peña, } \\
\text { Fdez-Riverola, \& Lourenço, } \\
\text { 2015) }\end{array}$ & 2014 & $\mathrm{R}$ & All type & $x$ & & & & $\times$ & & & $x$ \\
\hline 29 & Cliosoft dental & (“'Cliosoft dental,” 2014) & 2014 & $\mathrm{D}$ & Image & & & $\times$ & $x$ & & $x$ & & \\
\hline 30 & Medetect & (Tian et al., 2013) & 2013 & $\mathrm{D}$ & HTML & $x$ & & & & $x$ & & & $x$ \\
\hline 31 & Bioannote & (López-Fernández et al., 2013) & 2013 & $\mathrm{~B}$ & Image & $x$ & & & & $x$ & $x$ & & \\
\hline 32 & Biocat & (Zhou et al., 2013) & 2013 & $\mathrm{~B}$ & Image & $x$ & & & & $x$ & & $x$ & \\
\hline 33 & Gate & (Bontcheva et al., 2013) & 2013 & $\mathrm{~A}$ & Text & $x$ & & & $x$ & & $x$ & & $x$ \\
\hline 34 & BioQRator & (Kwon et al., 2013) & 2013 & $\mathrm{~A}$ & Text & & & $\times$ & $x$ & & & $x$ & \\
\hline 35 & Anafora & (Chen \& Styler, 2013) & 2013 & $\mathrm{~A}$ & Text & & $x$ & & $\times$ & & $x$ & $x$ & \\
\hline 36 & Ratsnake & (D.K., T., C., \& I., 2014) & 2012 & $\mathrm{R}$ & Image & & $x$ & & & $\times$ & & & $x$ \\
\hline 37 & Flersa & $\begin{array}{c}\text { (Navarro-Galindo \& Samos, } \\
\text { 2012) }\end{array}$ & 2012 & $\mathrm{R}$ & Image & $x$ & & & $x$ & & $x$ & & $x$ \\
\hline 38 & SMItag & $\begin{array}{l}\text { (Federico, Néstor, \& Oscar, } \\
\text { 2012) }\end{array}$ & 2012 & $\mathrm{R}$ & Image & & & $\times$ & & $\times$ & $\times$ & & \\
\hline 39 & Mammoapplet & (Mata et al., 2012) & 2012 & $\mathrm{R}$ & Image & & & $x$ & $x$ & & $x$ & & \\
\hline 40 & Brat & (Stenetorp et al., 2012) & 2012 & $\mathrm{~A}$ & Text & $x$ & & & $x$ & & $x$ & & \\
\hline 41 & Idash & (Ohno-Machado et al., 2012) & 2012 & $\mathrm{D}$ & Text & $x$ & & & $\times$ & & $x$ & & \\
\hline 42 & MedAt & (“Medat," 2011) & 2011 & $\mathrm{D}$ & Document & $x$ & & & & $x$ & & & $x$ \\
\hline 43 & DoctorEye & (David et al., 2012) & 2010 & $\mathrm{R}$ & Image & $x$ & & & $x$ & & $x$ & & $x$ \\
\hline 44 & Radsem & $\begin{array}{l}\text { (Möller, Regel, \& Sintek, } \\
\text { 2009) }\end{array}$ & 2009 & $\mathrm{D}, \mathrm{N}, \mathrm{R}$ & $\begin{array}{l}\text { Image, text, } \\
\text { video }\end{array}$ & & $x$ & & & $x$ & $\times$ & & \\
\hline 45 & @note & (“@ @note,” 2009) & 2009 & $\mathrm{~B}$ & text & $x$ & & & & $x$ & & $x$ & \\
\hline 46 & Ipad & $\begin{array}{c}\text { (Rubin, Rodriguez, Shah, \& } \\
\text { Beaulieu, 2008) }\end{array}$ & 2008 & $\mathrm{R}$ & Image & & $\times$ & & & $\times$ & $x$ & & \\
\hline
\end{tabular}




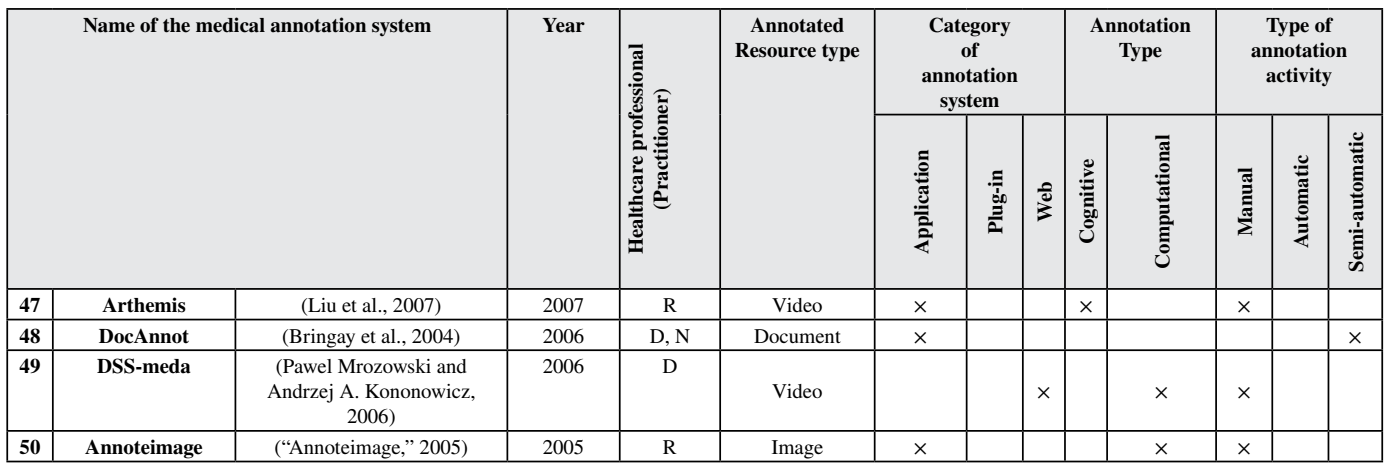

B: Biologist, D: Doctor, N: Nurse, R: Radiologist, A: all healthcare's professional

- Saving Annotation: Once the annotation is created it must be stored for the purpose of exploiting and reusing them. This function offers the possibility to record this annotation in a form specified by the constructor of the system;

- Delete Annotation: Deleting an annotation without archiving;

- Viewing Annotation: Many systems offer a specific view manner. These viewing methods include an ordering algorithm that offers the prioritization view, Gantt view... etc.;

- Sharing an Annotation: Share the annotation with healthcare professionals, this can help health professionals in collaboration;

- Send a Message: The PHC sends a document or record that contains annotations. This function assures asynchronous communication between PHC;

- Filtering Annotations: See below:

- Manual: The professional healthcare can choose to view only a collection of annotations chosen according to criteria;

- Automatic: The healthcare professional visualizes only the annotations for which helshe has the right to see;

- Searching Annotation: Searching an annotation based on several criteria;

- Fusing Annotated Documents: Creating a report of annotation with annotated documents. Fusing can offer a summary of the state of a patient based on annotation. Moreover, this function facilitates for paramedic practitioner the preparation of deposit report that is exchanged between paramedic healthcare which contains the state of all hospitalized patients;

- Comparing Annotation: This function allows us to compare annotations and decide if these annotations have the same significance or not;

- Refining Annotation: The practitioner draws a shape manually of an annotation object. Then, the computer intervenes automatically to re-traceladjust the shape drawn manually. This function used a lot in medical image annotation;

- Extracting Annotation: From an annotated document to be stored in a specific form (text, XML... etc.) or to be analyzed;

- Linking Annotation to an External Source: The annotated content is a link to an external source as a document, website...etc.;

- Localizing Annotation and Area Calculation of Annotated Zone: This function offers the coordinates of the shape used in the annotation and calculates the area of this shape. The medical image which has already been annotated can be reused with this function that is able to localize the annotation. The annotated zone in the image is an abnormal zone (sick zone) and the calculation of area can offer additional information; 
- Linking Annotations: This functionality offers the possibility of linking annotations. This link means that these annotations are dependent. Understanding an annotation is done by exploring other annotations related to this annotation;

- Classifying Annotations: This feature allows classifying annotations according to a classification parameter specified by the annotation system. The classification provides information that is easily exploited.

Table 2 presents a comparative study of the medical annotation systems seen in the literature review based on the functionalities offered by each system as a criterion for comparison. This technical study helps us in future works at the design of the medical annotation model.

\section{LIMITATIONS AND OBSERVATIONS}

The study of 50 annotation systems in the field of E-health, with the aim of providing a unified classification criteria and a comparison of the functionalities offered by these systems, allow us to deduce the following limitations and challenges:

\subsection{Lack of Liaison Between Resources Consulted and Annotations}

Several studies show that doctors use websites (Sophie clément, 2016). In 2010, according to the Center for Studies on Specialized Medical Information Materials, $76 \%$ of French generalist doctors used the Internet as part of their professional practice at least once a week (Teston, 2010). In Canada, in a study published in 2013, the interviewees used preferentially medical books or the opinion of a colleague to make a specific clinical decision. For continuing education, websites ranked third with $49 \%$ utilization, behind medical journals $93 \%$ and clinical practice guidelines $70 \%$ (Kosteniuk, Morgan, \& D'arcy, 2013). These external resources are consulted following a need for information during the identification and management of a health issue. The analysis of the information, also called the active reading of the information, provided by the patient is a combination of the reading with a certain critical thought, in this context a PHC can annotate the information of this document (Adler \& Van Doren, 2014). The annotations can take place on different resources and following these annotations the PHC tries to determine an action that is related to the patient health issue. The automatic link between the resources consulted and the annotations already made within these resources is a phase that is so far not done.

\subsection{Annotation is not an Objective}

The sharing of the same functionalities, already observed in Section 2.2, by medical annotation systems shows underuse of the semantics of medical annotative activity. This means that these systems provide the utilities to create an annotation and nothing beyond that. Annotating is not an objective, but it is a means to reach the goal (annotate to recall, annotate to explain, annotate to share, etc.). It can be said that the annotative activity begins with the creation of the annotation and ends with the invocation of the functionalities that can improve, assist and help the professional healthcare as soon as the annotation is created to benefit the most from it.

\subsection{Lack of the Specialized Systems of Annotation}

A patient record contains several resources (administrative, medical care, medical report...etc.). Each of these resources has a specificity related to several elements among which we quote: type of document, the staff of PHC working on this document. Indeed, these two elements were used as classification criteria in table1. The specificity of each medical document requires the existence of a specific annotation tool, which uses a specific dictionary of word used by this PHC, for each type of document. This study shows that most existing medical annotation systems are developed to annotate 
Table 2. Comparative study of the medical annotation systems using the functionalities offered by each system

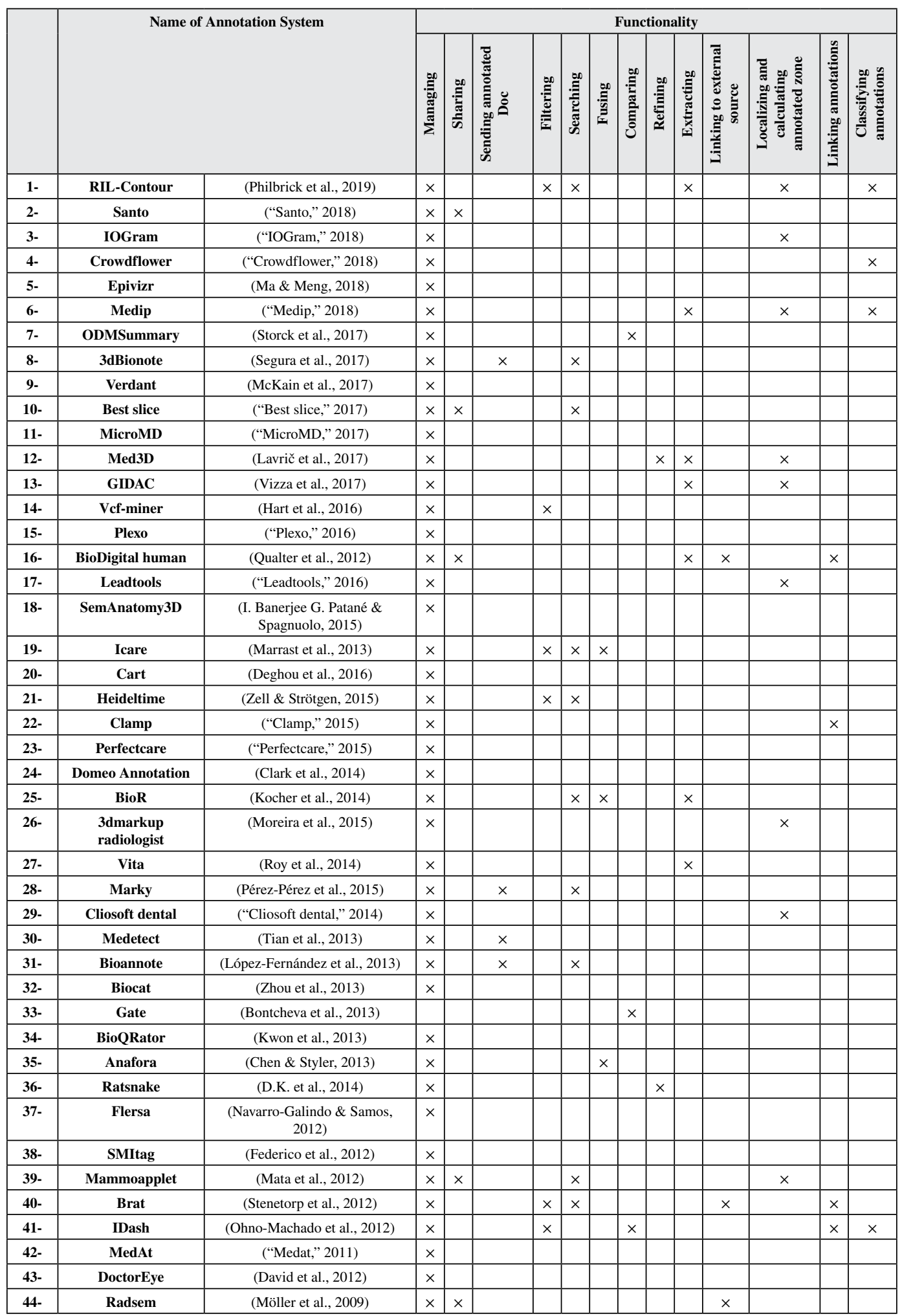




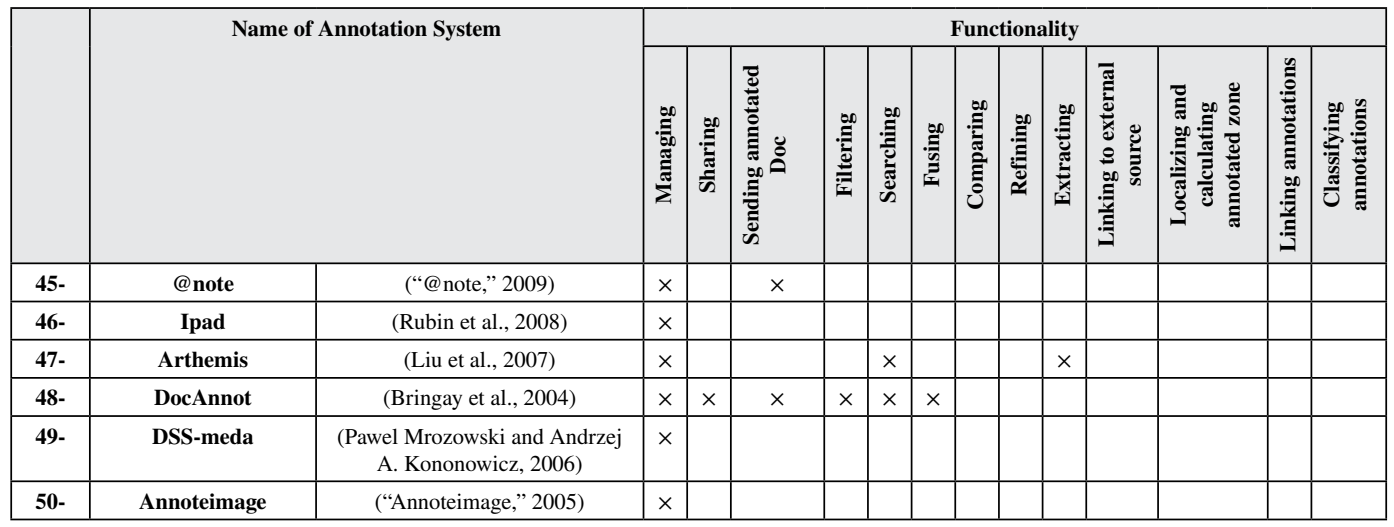

biomedical documents, medical imaging, and natural language written in medical documents (Natural Language Processing) to allow the PHC to collaborate with others PHC, while a medical record is composed of several resources that are not fully covered by specific annotation systems.

\subsection{Lack of Modeling a Healthcare Cycle}

The healthcare cycle consists of 4 phases: diagnosis, treatment, advice, follow-up, and observation. For a treatment, that is phase 2 of the healthcare cycle, a patient may have one or more cycles of treatment that are strongly linked together to help a PHC in the identification and management of a health problem. But what we have seen in these systems that treat the problem of annotation in the electronic health record (Bringay et al., 2004), (Marrast et al., 2013), there is no consideration of this cycle of healthcare. For example, authors in (Bringay et al., 2004) discussed the possibility of collaboration between PHC to avoid lexical disambiguation, using speech acts, they did not propose a healthcare cycle. The collaboration between the PHC should be directed to the implementation of a conceptual model that considers the creation of the healthcare cycle. As a result of the implementation of the healthcare cycle, the annotations are arranged by phases, linked together in the same phase (as the 2 cycles of treatment) or inter-phase, organized in a way that reflects the creative logic of these annotations. This organization can be a step towards setting up a communication platform with existing annotation systems.

\subsection{Lack of the Competency Modeling of PHC}

The annotation can be created by a PHC who admits a certain competence in his field. The competence of an individual, at the general sense of the term, is its ability to combine and mobilize through resources such the medical knowledge helshe has extra documents...etc. to identify new solutions or to understand more about certain situations. The available annotation systems do not provide the recommended resources to the PHC. This is because those systems do not have the ability to understand the meaning of the information being annotated and do not track how PHC treats the medical record (absence of healthcare cycle). In other words, the system is not intelligent to mimic PHC competency modellbehavior. Therefore, modeling PHC competency will eventually help in creating intelligent annotation systems.

\subsection{The Problem of Interoperability}

We have seen in this study a fragmented image of annotation systems. Each annotation system is developed by a programming language and each of these uses a different medical dictionary to 
annotate resources. The negative effect of this image is seen technically by the difficulty of ensuring interoperability between annotation systems in the digital health field. Interoperability is defined by the ability to cooperate and exchange data despite differences in languages, interface, and execution platform (Heubusch, 2006), (Iroju, Soriyan, Gambo, \& Olaleke, 2013). The interoperability of medical annotation systems and even medical information systems, which intervene in healthcare, is a major challenge but there are some obstacles that paralyze the application of this notion that has direct positive effects on human health. The Major obstacles to the application of interoperability of medical annotation systems:

- The lack of a computer infrastructure that allows the exchange of healthcare data between the PHC of private sectors and between the PHC of the private sector and public sectors;

- The complexity of the health field contains a multi-practitioner (nurses, radiologists, physicians, biologists, etc.) who work together to provide patient care. The exchange of information between these practitioners must retain the information as it is provided by ensuring the integrity of the information and the security of data exchanged (Bai, Dai, \& Li, 2014; Ryan, 2006). An error that comes from the exchange of this information can lead to the death of the patient or privacy issue;

- Several standards that are created (DICOM, ICD, HL7, OpenEHR, etc.) and used in medical annotation systems. This varied use of standards also the non-normalization of abbreviations used by PHCs makes the application of the interoperability difficult. This problem of not standardizing the used abbreviations leads to the problem of incompatible ontologies and terminology dictionaries (Lau \& Shakib, 2005).

\section{CONCLUSION AND FUTURE WORK}

In this paper, we studied annotation systems of the digital health domain available in industrial and research areas in order to propose a unified classification of this kind of system that is omnipresent in hospital information systems. This panoramic view provided is based on the classification of fifty different annotation systems developed in the literature over the past two decades. The presented list of annotation systems is not exhaustive, but it contains the majority of annotation systems encountered in our survey of annotation tools. Even if there are other annotation systems developed in the literature which is not mentioned in this article, it is certain that these systems can be easily integrated into our classification since the categorization technique is based on cross-cutting criteria applicable for any annotation system that is not exist in our proposed list. The proposed organization of annotation tools is built on the basis of five criteria. Moreover, we classified the annotation systems according to the functionalities each one provides. This classification based on criteria, already explained in our study, which are transversal organizational criteria, facilitates the identification of limitations and possible challenges in the area of the medical annotation systems. In future research, we try to use the results of this study to create an annotation ontological model for PHCs and then try to generalize them to be functional for all professionals in different domains. Based on the model we are going to propose an annotation application that overcomes the shortcomings raised by this research. This application will offer a list of services that can assist the PHC based on the semantics of the annotation made by the latter and on intelligent reasoning mechanisms. 


\section{REFERENCES}

Adler, M. J., \& Van Doren, C. (2014). How to read a book: The classic guide to intelligent reading. Simon and Schuster.

Annoteimage. (2005). Retrieved from http://www.si.washington.edu/content/annoteimage

Azouaou, F. (2006). Modèles et outils d'annotations pour une mémoire personnelle de l'enseignant. Université Joseph-Fourier-Grenoble I.

Bai, Y., Dai, L., \& Li, J. (2014). Issues and Challenges in Securing eHealth Systems. International Journal of E-Health and Medical Communications, 5(1), 1-19. doi:10.4018/ijehmc.2014010101

Banerjee, I., Patané, G., \& Spagnuolo, M. (2015). SemAnatomy3D: Annotation of patient-specific anatomy. In Smart Tools and Apps for Graphics - Eurographics Italian Chapter Conference. doi:<ALIGNMENT.qj ></ ALIGNMENT>10.13140/RG.2.1.4696.7762

Best slice. (2017). Retrieved from https://www.best.edu.au/s/5njv62ar?data=8\%400!9\%4028320!10\%4027251.5\&version $=1$

Bontcheva, K., Cunningham, H., Roberts, I., Roberts, A., Tablan, V., Aswani, N., \& Gorrell, G. (2013). GATE Teamware: A web-based, collaborative text annotation framework. Language Resources and Evaluation, 47(4), 1007-1029. doi:10.1007/s10579-013-9215-6

Bringay, S., Barry, C., \& Charlet, J. (2004). Les documents et les annotations du dossier patient hospitalier. Inormation - Interaction - Intelligence.

Cahier, J. P., \& Zacklad, M. (2004). Socio-Semantic Web'’ applications: Towards a methodology based on the Theory of the Communities of Action. Resource (Saint Joseph, Mich.).

Charlet, J., Bachimont, B., Brunie, V., el Kassar, S., Zweigenbaum, P., \& Boisvieux, J. F. (1998). Hospitexte: Towards a document-based hypertextual electronic medical record. Proc AMIA Symp, 713. PMID:9929312

Chehab, K., Kalboussi, A., \& Kacem, A. H. (2018). Study of annotations in e-health domain. In Lecture Notes in Computer Science (including subseries Lecture Notes in Artificial Intelligence and Lecture Notes in Bioinformatics) (Vol. 10898). doi:10.1007/978-3-319-94523-1_17

Chehab, K., Kalboussi, A., \& Kacem, A. H. (2019). An Annotation Model for Patient Record. In HEALTHINF (pp. 272-280). Academic Press.

Chehab, K., Kalboussi, A., \& Kacem, A. H. (2020). Study of Healthcare Professionals' Interaction in the Patient Records Based on Annotations. In International Conference on Smart Homes and Health Telematics (pp. 316-328). doi:10.1007/978-3-030-51517-1_28

Chen, W.-T., \& Styler, W. (2013). Anafora: a web-based general purpose annotation tool. In Proceedings of the conference. Association for Computational Linguistics. North American Chapter. Meeting (Vol. 2013, p. 14). NIH Public Access.

Clamp. (2015). Retrieved from https://clamp.uth.edu/res/CLAMP-Manual7_5_17.pdf

Clark, T., Ciccarese, P. N., \& Goble, C. A. (2014). Micropublications: A semantic model for claims, evidence, arguments and annotations in biomedical communications. Journal of Biomedical Semantics, 5(1), 28. doi:10.1186/2041-1480-5-28 PMID:26261718

Clean tools. (2018). Retrieved from https://arxiv.org/ftp/arxiv/papers/1808/1808.03806.pdf

Cliosoft dental. (2014). Retrieved from https://sotaimaging.com

Colley, A., Rantakari, J., Virtanen, L., \& Häkkilä, J. (2017). Mediating Interaction Between Healthcare Professionals and Patients with a Dual-Sided Tablet. In IFIP Conference on Human-Computer Interaction (pp. 54-61). Springer. doi:10.1007/978-3-319-67687-6_4

Crowdflower. (2018). Retrieved from https://www.ncbi.nlm.nih.gov/pmc/articles/PMC4299942 
David, R., Graf, N., Karatzanis, I., Stenzhorn, H., Manikis, G., \& Sakkalis, V., .. Marias, K. (2012). Clinical evaluation of DoctorEye platform in nephroblastoma. In Proceedings of the 2012 th International Advanced Research Workshop on In Silico Oncology and Cancer Investigation - The TUMOR Project Workshop, IARWISOCI 2012 (pp. 1-4). Academic Press.

Deghou, S., Zeller, G., Iskar, M., Driessen, M., Castillo, M., Van Noort, V., \& Bork, P. (2016). CART - A chemical annotation retrieval toolkit. Bioinformatics (Oxford, England), 32(18), 2869-2871. doi:10.1093/ bioinformatics/btw233 PMID:27256313

Denoue, L. (2000). De la création à la capitalisation des annotations dans un espace personnel d'informations. Academic Press.

Dingli, A., \& Seychell, D. (2014). Using \{RFID\} and Wi-Fi in Healthcare. International Journal of E-Health and Medical Communications, 5(1), 96-113. doi:10.4018/ijehmc.2014010106

D.K., I., T., G., C., S., \& I., M. (2014). Ratsnake: A versatile image annotation tool with application to computeraided diagnosis. TheScientificWorldJournal, 8(1), 73-126. doi:10.1155/2014/286856

Dutta, N., Mondal, A., \& Paul, P. (2020). An Annotation System to Annotate Healthcare Information from Tweets. In Emerging Technology in Modelling and Graphics (pp. 319-325). Springer.

Federico, L., Néstor, D., \& Oscar, C. (2012). SMITag: A social network for semantic annotation of medical images. In 38th Latin America Conference on Informatics, CLEI 2012 - Conference Proceedings (pp. 1-7). doi:10.1109/CLEI.2012.6427187

Hart, S. N., Duffy, P., Quest, D. J., Hossain, A., Meiners, M. A., \& Kocher, J. P. (2016). VCF-Miner: GUI-based application for mining variants and annotations stored in VCF files. Briefings in Bioinformatics, 17(2), 346-351. doi:10.1093/bib/bbv051 PMID:26210358

Heubusch, K. (2006). Interoperability: What it means, why it matters. Journal of American Health Information Management Association, 77(1), 26-30. PMID:16475733

Huart, P. (1996). Définition d'un poste de lecture active de documents électroniques. Academic Press.

IOGram. (2018). Retrieved from http://iogram.ca

Iroju, O., Soriyan, A., Gambo, I., \& Olaleke, J. (2013). Interoperability in healthcare: Benefits, challenges and resolutions. International Journal of Innovation and Applied Studies, 3(1), 262-270.

Kahan, J., \& Koivunen, M.-R. (2001). Annotea: an open RDF infrastructure for shared Web annotations. In Proceedings of the 10th international conference on World Wide Web (pp. 623-632). doi:10.1145/371920.372166

Kalboussi, A., Mazhoud, O., Hadj Kacem, A., \& Omheni, N. (2013). A formal model of learner's annotations dedicated to web services invocation. In Proceedings of the 21st International Conference on Computers in Education, ICCE 2013 (pp. 166-169). Academic Press.

Kalboussi, A., Mazhoud, O., \& Kacem, A. H. (2013). Annotative Activity as a Potential Source of Web Service Invocation. <ALIGNMENT.qj></ALIGNMENT>10.5220/0004369002880292

Kalboussi, A., Mazhoud, O., \& Kacem, A. H. (2016a). Comparative study of web annotation systems used by learners to enhance educational practices: Features and services. International Journal of Technology Enhanced Learning, 8(2), 129-150. doi:10.1504/IJTEL.2016.078081

Kalboussi, A., Mazhoud, O., \& Kacem, A. H. (2016b). Functionalities provided by annotation systems for learners in educational context: An overview. International Journal of Emerging Technologies in Learning, 11(2), 4-11. doi:10.3991/ijet.v11i02.5166

Kalboussi, A., Mazhoud, O., Omheni, N., \& Kacem, A. H. (2014). A new annotation system based on a semantic analysis of a learner's annotative activity to invoke web services. International Journal of Metadata, Semantics and Ontologies, 9(4), 350-370. doi:10.1504/IJMSO.2014.065447

Kalboussi, A., Omheni, N., Mazhoud, O., \& Kacem, A. H. (2015a). An interactive annotation system to support the learner with web services assistance. In 2015 IEEE 15th International Conference on Advanced Learning Technologies (pp. 409-410). IEEE. doi:10.1109/ICALT.2015.57 
Kalboussi, A., Omheni, N., Mazhoud, O., \& Kacem, A. H. (2015b). How to organize the annotation systems in human-computer environment: Study, classification and observations. In Lecture Notes in Computer Science (including subseries Lecture Notes in Artificial Intelligence and Lecture Notes in Bioinformatics) (pp. 115-133). doi:<ALIGNMENT.qj></ALIGNMENT>10.1007/978-3-319-22668-2_11

Kocher, J. P. A., Quest, D. J., Duffy, P., Meiners, M. A., Moore, R. M., Rider, D., Hossain, A., Hart, S. N., \& Dinu, V. (2014). The Biological Reference Repository (BioR): A rapid and flexible system for genomics annotation. Bioinformatics (Oxford, England), 30(13), 1920-1922. Advance online publication. doi:10.1093/ bioinformatics/btu137 PMID:24618464

Kosteniuk, J. G., Morgan, D. G., \& D'arcy, C. K. (2013). Use and perceptions of information among family physicians: Sources considered accessible, relevant, and reliable. Journal of the Medical Library Association: JMLA, 101(1), 32-37. doi:10.3163/1536-5050.101.1.006 PMID:23405045

Kraljevic, Z., Bean, D., Mascio, A., Roguski, L., Folarin, A., Roberts, A., ... Dobson, R. (2019). MedCAT-Medical Concept Annotation Tool. ArXiv Preprint ArXiv:1912.10166

Kwon, D., Kim, S., Shin, S.-Y., \& Wilbur, W. J. (2013). BioQRator: a web-based interactive biomedical literature curating system. In Fourth BioCreative Challenge Workshop (pp. 241-246). Academic Press.

Lau, L. M., \& Shakib, S. (2005). Towards Data Interoperability: Practical Issues in Terminology Implementation and Mapping. HIC 2005 and HINZ 2005: Proceedings, 208.

Lavrič, P., Bohak, C., \& Marolt, M. (2017). Collaborative view-aligned annotations in web-based 3D medical data visualization. In 2017 40th International Convention on Information and Communication Technology, Electronics and Microelectronics, MIPRO 2017 - Proceedings (pp. 259-263). doi:<ALIGNMENT.qj $><1$ ALIGNMENT > 10.23919/MIPRO.2017.7973430

Leadtools. (2016). Retrieved from https://www.leadtools.com/sdk/annotation

Liu, D., Cao, Y., Kim, K. H., Stanek, S., Doungratanaex-Chai, B., Lin, K., Tavanapong, W., Wong, J., Oh, J. H., \& de Groen, P. C. (2007). Arthemis: Annotation software in an integrated capturing and analysis system for colonoscopy. Computer Methods and Programs in Biomedicine, 88(2), 152-163. doi:10.1016/j.cmpb.2007.07.011 PMID: 17854947

López-Fernández, H., Reboiro-Jato, M., Glez-Peña, D., Aparicio, F., Gachet, D., Buenaga, M., \& Fdez-Riverola, F. (2013). BioAnnote: A software platform for annotating biomedical documents with application in medical learning environments. Computer Methods and Programs in Biomedicine, 111(1), 139-147. doi:10.1016/j. cmpb.2013.03.007 PMID:23562645

Ma, J., \& Meng, J. (2018). Interactive genomic visualization for R/bioconductor. In BIBE 2018; International Conference on Biological Information and Biomedical Engineering (pp. 1-4). VDE.

Marrast, P., \& Zaraté, P., \& Mayère, a. (2013). How to support coordination through annotations?: a longitudinal case study of nurses' work in an oncology hospital. Proceedings of the 2013 Conference on Computer Supported Cooperative Work Companion - CSCW'13. doi:10.1145/2441955.2442007

Marshall, C. (1997). Annotation: from paper books to the digital library. In Proceedings of the second ACM international conference on Digital libraries. (pp. 131-140). ACM Press. doi:10.1145/263690.263806

Mata, C., Oliver, A., Torrent, A., \& Martí, J. (2012). MammoApplet: An interactive Java applet tool for manual annotation in medical imaging. In IEEE 12th International Conference on BioInformatics and BioEngineering, BIBE 2012 (pp. 34-39). doi:<ALIGNMENT.qj></ALIGNMENT>10.1109/BIBE.2012.6399703

McKain, M. R., Hartsock, R. H., Wohl, M. M., \& Kellogg, E. A. (2017). Verdant: Automated annotation, alignment and phylogenetic analysis of whole chloroplast genomes. Bioinformatics (Oxford, England), 33(1), 130-132. doi:10.1093/bioinformatics/btw583 PMID:27634949

Medat. (2011). Retrieved from https://nit.felk.cvut.cz/drupal/projects/medical-annotation-tool-medat

Medip. (2018). Retrieved from http://www.medicalip.com/en/

MicroM. D. (2017). Retrieved from https://www.micromd.com/emr/ 
Mille, D. (2005). Modèles et outils logiciels pour l'annotation sémantique de documents pédagogiques. Joseph Fourier University. Retrieved from https://tel.archives-ouvertes.fr/tel-00011468

Möller, M., Regel, S., \& Sintek, M. (2009). RadSem: Semantic annotation and retrieval for medical images. In Lecture Notes in Computer Science (including subseries Lecture Notes in Artificial Intelligence and Lecture Notes in Bioinformatics) (pp. 21-35). doi:<ALIGNMENT.qj></ALIGNMENT>10.1007/978-3-642-02121-3_6

Moreira, D. A., Hage, C., Luque, E. F., Willrett, D., \& Rubin, D. L. (2015). 3D markup of radiological images in ePAD, a web-based image annotation tool. In Proceedings - IEEE Symposium on Computer-Based Medical Systems (pp. 97-102). doi:<ALIGNMENT.qj></ALIGNMENT>10.1109/CBMS.2015.46

Navarro-Galindo, J. L., \& Samos, J. (2012). The FLERSA tool: Adding semantics to a web content management system. International Journal of Web Information Systems. <ALIGNMENT.qj $></$ ALIGNMENT > 10.1108/17440081211222609

@ note. (2009). Retrieved from http://anote-project.org

Ohno-Machado, L., Bafna, V., Boxwala, A. A., Chapman, B. E., Chapman, W. W., Chaudhuri, K., \& Zambon, A. et al. (2012). iDASH: Integrating data for analysis, anonymization, and sharing. Journal of the American Medical Informatics Association, 19(2), 196-201. doi:10.1136/amiajnl-2011-000538 PMID:22081224

Pawel Mrozowski and Andrzej A. Kononowicz. (2006). DSS-MEDA: A web-based framework for video annotation in medical e-learning. Bio-Algorithms and Med-Systems, 2(4), 51-56. Retrieved from https://dblp. org/rec/bib/journals/bams/MrozowskiK06

Pérez-Pérez, M., Glez-Peña, D., Fdez-Riverola, F., \& Lourenço, A. (2015). Marky: A tool supporting annotation consistency in multi-user and iterative document annotation projects. Computer Methods and Programs in Biomedicine, 118(2), 242-251. doi:10.1016/j.cmpb.2014.11.005 PMID:25480679

Perfectcare. (2015). Retrieved from https://www.ncgmedical.com/practice-software-solutions/electronic-medicalrecords/

Philbrick, K. A., Weston, A. D., Akkus, Z., Kline, T. L., Korfiatis, P., Sakinis, T., Kostandy, P., Boonrod, A., Zeinoddini, A., Takahashi, N., \& Erickson, B. J. (2019). RIL-contour: A medical imaging dataset annotation tool for and with deep learning. Journal of Digital Imaging, 32(4), 571-581. doi:10.1007/s10278-019-00232-0 PMID:31089974

Plexo. (2016). Retrieved from https://diegocantor.com/projects/

Qualter, J., Sculli, F., Oliker, A., Napier, Z., Lee, S., \& Garcia, J. ... Triola, M. (2012). The BioDigital human: A web-based 3D platform for medical visualization and education. In Studies in Health Technology and Informatics. doi:<ALIGNMENT.qj></ALIGNMENT>10.3233/978-1-61499-022-2-359

Roy, S., Brown, M. S., \& Shih, G. L. (2014). Visual interpretation with three-dimensional annotations (VITA): Three-dimensional image interpretation tool for radiological reporting. Journal of Digital Imaging, $27(1)$, 49-57. doi:10.1007/s10278-013-9624-5 PMID:23979113

Rubin, D. L., Rodriguez, C., Shah, P., \& Beaulieu, C. (2008). iPad: Semantic annotation and markup of radiological images. In AMIA Annu Symp Proc. (p. 626). Academic Press.

Ryan, A. (2006). Towards semantic interoperability in healthcare: ontology mapping from SNOMED-CT to HL7 version 3. In Proceedings of the second Australasian workshop on Advances in ontologies-Volume 72 (pp. 69-74). Academic Press.

Santo. (2018). Retrieved from http://aclweb.org/anthology/P18-4012

Segura, J., Sanchez-Garcia, R., Martinez, M., Cuenca-Alba, J., Tabas-Madrid, D., Sorzano, C. O. S., \& Carazo, J. M. (2017). 3DBIONOTES v2.0: A web server for the automatic annotation of macromolecular structures. Bioinformatics (Oxford, England), 33(22), 3655-3657. doi:10.1093/bioinformatics/btx483 PMID:28961691

Sophie Clément, P. (2016). Quels sites internet utilisent les médecins généralistes en consultation? Academic Press. 
Stenetorp, P., Pyysalo, S., Topic, G., Ohta, T., Ananiadou, S., \& Tsujii, J. (2012). BRAT: a Web-based Tool for NLP-Assisted Text Annotation. In 13th Conference of the European Chapter of the ACL (pp. 102-107). doi:<ALIGNMENT.qj></ALIGNMENT>10.1007/s41465-017-0039-y

Storck, M., Krumm, R., \& Dugas, M. (2017). ODMSummary: A tool for automatic structured comparison of multiple medical forms based on semantic annotation with the Unified Medical Language System. PLoS One, 11(10), e0164569. doi:10.1371/journal.pone.0164569 PMID:27736972

Teston, R. (2010). L'utilisation d'internet se consolide chez les professionnels de santé. Retrieved from https:// buzz-esante.com/tag/cessim/

Tian, L., Zhang, W., Bikakis, A., Wang, H., Yu, Y., Ni, Y., \& Cao, F. (2013). MeDetect: A LOD-based system for collective entity annotation in Biomedicine. In Proceedings - 2013 IEEE/WIC/ACM International Conference on Web Intelligence, WI 2013 (pp. 233-240). doi:<ALIGNMENT.qj></ALIGNMENT>10.1109/WI-IAT.2013.34

Veron, M. (1997). Modélisation de la composante annotative dans les documents électroniques. Academic Press.

Vizza, P., Guzzi, P. H., Veltri, P., Cascini, G. L., Curia, R., \& Sisca, L. (2017). GIDAC: A prototype for bioimages annotation and clinical data integration. In Proceedings - 2016 IEEE International Conference on Bioinformatics and Biomedicine, BIBM 2016 (pp. 1028-1031). doi:<ALIGNMENT.qj></ALIGNMENT>10.1109/ BIBM.2016.7822663

Weibel, S. (1997). The Dublin Core: A simple content description model for electronic resources. Bulletin of the American Society for Information Science and Technology, 24(1), 9-11. doi:10.1002/bult.70

Yu, W. D., \& Yilayavilli, S. K. (2010). A Semantic-Based Dynamic Search Engine Design and Implementation for Electronic Medical Records. International Journal of E-Health and Medical Communications, 1(2), 61-73. doi:10.4018/jehmc.2010040106

Zell, J., \& Strötgen, J. (2015). HeidelTime Standalone Manual Version 2.1. Proceedings of the 5th International Workshop on Semantic Evaluation.

Zhou, J., Lamichhane, S., Sterne, G., Ye, B., \& Peng, H. (2013). BIOCAT: A pattern recognition platform for customizable biological image classification and annotation. BMC Bioinformatics, 14(1), 291. doi:10.1186/14712105-14-291 PMID:24090164

Khalil Chehab received his Master's degree in Computer Science in 2015 from the Higher Institute of Computer Science and Management of Kairouan. He is a member of the ReDCAD Research Laboratory. Since 2018, he is a PhD student at the University of Sfax. His PhD subject focuses on the analysis of the semantics of the healthcare Professional annotative activity.

Anis Kalboussi is an Associate Professor in Computer Science at the Kairouan University. He received his Master's Degree and his PhD in Computer Science respectively from the University of Kairouan in 2011 and from the University of Sfax in 2015. Since 2018, he is the Head of the Computer Science Department at the Higher Institute of Computer Science and Management of Kairouan University. He is a member of the ReDCAD Research Laboratory at the National School of Engineers of Sfax.

Ahmed Hadj Kacem received his MS in Computer Science and his PhD in Computer Science from UPS, University Paul Sabatier Toulouse-III, respectively in 1991 and 1995. He joined the University of Sfax (Tunisia) as an Assistant Professor in 1997, then as a full Professor in 2007. Since 2014, he has been the Dean of the Faculty of Economics and Management of Sfax. He is a member of the ReDCAD Research Laboratory. He is an IEEE senior member and he is founder and chair of the Computer Society Chapter during 2009. 